\title{
A PRACTICAL ADAPTIVE BLIND MULTICHANNEL ESTIMATION ALGORITHM WITH APPLICATION TO ACOUSTIC IMPULSE RESPONSES
}

\author{
Rehan Ahmad, Andy W. H. Khong and Patrick A. Naylor \\ Imperial College London \\ Department of Electrical and Electronic Engineering \\ \{rehan.ahmad02, andy.khong, p.naylor\}@imperial.ac.uk
}

\begin{abstract}
We propose a noise robust adaptive blind multichannel identification algorithm for acoustic impulse responses. It has been known that the normalized multichannel frequency domain least-meansquare (NMCFLMS) algorithm misconverges under low signal-tonoise ratio. The coefficients of NMCFLMS converge initially toward the true impulse response after which they then misconverge. The extended NMCFLMS (ext-NMCFLMS) algorithm which has been proposed to mitigate this misconvergence problem assumes the knowledge of magnitude and time-differences-of-arrival (TDOA) of the direct paths for the acoustic impulse responses. In this work, we show how the TDOA can be obtained. More importantly, we present a novel approach to estimate the magnitude of the direct path component under practical conditions. We then show how these estimates can be incorporated to the proposed ext-NMCFLMS with direct path estimation algorithm. We analyze how errors in these estimates affect the performance of the proposed algorithm.
\end{abstract}

Index Terms - Blind channel identification, adaptive signal processing, time-delay estimation

\section{INTRODUCTION}

The profound interest in blind channel identification (BCI) techniques in recent years arise due to their extensive applications in signal processing. For applications such as speech dereverberation for example, the identified acoustic channel can be utilized, after inversion, to remove the degradation introduced by the acoustic channels. Techniques for BCI can generally be classified into two main classes (a) higher order statistical (HOS) and (b) second order statistical (SOS) methods. Although HOS methods [1] were proposed for $\mathrm{BCI}$ due to the rich information, large number of observation samples are required and, in addition, these methods suffer from a slow rate of convergence. As a result, SOS methods such as [2] [3] have become more popular. These methods utilize the cross relation (CR) equality between the channels and the observed channel outputs. Subspace methods [4] offer another alternative. These methods estimate the channel parameters through subspace decomposition of the received data matrix utilizing the principle of orthogonality between the signal and noise subspaces. Comparisons between SOS and HOS methods have been presented in [5].

More recently, adaptive BCI techniques have been proposed. The normalized multichannel frequency domain least-meansquare (NMCFLMS) algorithm [6] has been shown to be effective in identifying room impulse responses for acoustic dereverberation. This algorithm is derived by iteratively minimizing the cost function defined as the sum square errors between the interchannel crosscorrelation of a microphone array.

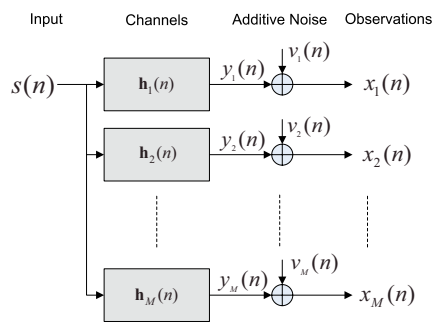

Fig. 1. Relationship between input and output in a SIMO model.

One of the main challenges for NMCFLMS is that the algorithm suffers from a misconvergence problem [7]. It has been shown through simulations presented in [8] [9] [10] that the estimated filter coefficients converge first toward the impulse response of the acoustic system but then misconverge. Under low signal-to-noise ratio (SNR) conditions, the effect of misconvergence becomes more significant and occurs at an earlier stage of adaptation.

To mitigate this misconvergence problem, the extendedNMCFLMS (ext-NMCFLMS) algorithm [8] is proposed where the direct path component of each channel is assumed to be known while the remaining filter coefficients are estimated using the NMCFLMS algorithm [6]. This a priori knowledge of the direct path components includes (a) their magnitude and (b) their time-differencesof-arrival (TDOA) for each channel. In this paper, we propose a novel practical approach to avoid misconvergence under low SNR. The proposed extended NMCFLMS with direct path estimation algorithm (ext-NMCFLMS ${ }_{\mathrm{DPE}}$ ) employs the GCC algorithm [11] to estimate the TDOA of the direct path components. More importantly, we propose a novel method to estimate the magnitude of the direct path components. We investigate how estimation errors for the time-delay and magnitude affect the performance of the proposed ext-NMCFLMS $S_{\text {DPE }}$ algorithm. We show the robustness of the ext-NMCFLMS $S_{\mathrm{DPE}}$ algorithm through simulation examples using both white Gaussian noise (WGN) and speech sequences.

\section{ADAPTIVE BCI ALGORITHMS}

\subsection{Problem statement}

We consider a single-input multiple-output (SIMO) finite impulse response linear system as shown in Fig. 1. The $i$ th channel output signal $x_{i}(n)$ is given by

$$
\mathbf{x}_{i}(n)=\mathbf{H}_{i}(n) \mathbf{s}(n)+\mathbf{v}_{i}(n)
$$


for $i=1,2, \ldots, M$, where $M$ is the number of channels, $\mathbf{x}_{i}(n)=\left[\begin{array}{llll}x_{i}(n) & x_{i}(n-1) & \ldots & x_{i}(n-L+1)\end{array}\right]^{T}$, $\mathbf{h}_{i}(n)=\left[h_{i, 0}(n) h_{i, 1}(n) \ldots h_{i, L-1}(n)\right]^{T}, \mathbf{s}(n)=[s(n) s(n-$ 1) $\ldots s(n-2 L+2)]^{T}, \mathbf{v}_{i}(n)=\left[v_{i}(n) v_{i}(n-1) \ldots v_{i}(n-L+\right.$ $1)]^{T}$,

$$
\mathbf{H}_{i}(n)=\left[\begin{array}{ccccc}
h_{i, 0}(n) & \cdots & h_{i, L-1}(n) & \cdots & 0 \\
\vdots & \ddots & \vdots & \ddots & \vdots \\
0 & \cdots & h_{i, 0}(n) & \cdots & h_{i, L-1}(n)
\end{array}\right]
$$

while $L$ is the length of the longest impulse response and superscript ${ }^{T}$ denotes transposition. We assume that the additive noise on $M$ channels is uncorrelated, i.e., $E\left\{v_{i}(n) v_{j}(n)\right\}=0$ for $i \neq j$ and $E\left\{v_{i}(n) v_{i}\left(n-n^{\prime}\right)\right\}=0$ for $n \neq n^{\prime}$ while $E\left\{v_{i}(n) s(n)\right\}=0$. For channel identifiability [3], we also assume that the channel transfer function does not contain any common zeros and the autocorrelation matrix of the source signal $\mathbf{R}_{s s}(n)=E\left\{s(n) s^{T}(n)\right\}$ is full rank.

A blind multichannel system can be identified, in the absence of noise, using the cross-relationship between the $i$ th and $j$ th channel outputs given [6], for $i \neq j$, by $\mathbf{x}_{i}^{T}(n) \mathbf{h}_{j}(n)=\mathbf{x}_{j}^{T}(n) \mathbf{h}_{i}(n)$ for $i, j=1, \ldots, M$. An a priori error exists if noise is present, or the channels are estimated with error given, for $i \neq j$, by

$$
e_{i j}(n)=\mathbf{x}_{i}^{T}(n) \widehat{\mathbf{h}}_{j}(n-1)-\mathbf{x}_{j}^{T}(n) \widehat{\mathbf{h}}_{i}(n-1),
$$

where $\widehat{\mathbf{h}}_{i}(n)$ is the estimated $i$ th channel impulse response. Using (3), adaptive BCI algorithms such as NMCFLMS are derived by minimizing the cost function

$$
J(n)=\frac{1}{\|\widehat{\mathbf{h}}(n)\|_{2}^{2}} \sum_{i=1}^{M-1} \sum_{j=i+1}^{M} e_{i j}^{2}(n)
$$

with respect to the estimated impulse response $\widehat{\mathbf{h}}_{i}(n)$ for $i=$ $1, \ldots, M$ where $\widehat{\mathbf{h}}=\left[\widehat{\mathbf{h}}_{1}^{T} \ldots \widehat{\mathbf{h}}_{M}^{T}\right]^{T}$ and $\|\cdot\|_{2}^{2}$ denotes squared $l_{2}$ norm. The NMCFLMS [6] algorithm is given, for each $m$ th frame, by:

$$
\begin{aligned}
\underline{\epsilon}_{i j}^{01}(m)= & \mathcal{W}_{L \times 2 L}^{01}\left[\mathcal{D}_{x_{i}}(m) \mathcal{W}_{2 L \times L}^{10} \underline{\widehat{\mathbf{h}}}_{j}(m)-\mathcal{D}_{x_{j}}(m) \mathcal{W}_{2 L \times L}^{10} \underline{\widehat{\mathbf{h}}}_{i}(m)\right], \\
\mathcal{P}_{i}(m)= & \lambda \mathcal{P}_{i}(m-1)+(1-\lambda) \sum_{j=1, j \neq i}^{M} \mathcal{D}_{x_{j}}^{*}(m) \mathcal{D}_{x_{j}}(m), \\
\underline{\underline{\mathbf{h}}}_{i}^{10}(m)= & \hat{\underline{\mathbf{h}}}_{i}^{10}(m-1)-\rho\left[\mathcal{P}_{i}(m)+\delta \mathbf{I}_{2 L \times 2 L}\right]^{-1} \times \\
& \sum_{j=1}^{M} \mathcal{D}_{x_{j}}^{*}(m) \underline{\epsilon}_{j i}^{01}(m), \quad i=1, \ldots, M,
\end{aligned}
$$

where ${ }^{*}$ denotes complex conjugate, $0<\rho \leq 1$ is the step-size, $\lambda=[1-1 /(3 L)]^{L}$ is the forgetting factor and $\delta$ is the small regularization constant. We have also denoted $\mathbf{I}_{L \times L}, \mathbf{0}_{L \times L}$, and $\mathbf{F}_{L}$ as the identity, null and Fourier matrices of dimension $L \times L$ respectively, $\mathbf{W}_{L \times 2 L}^{01}=\left[\mathbf{0}_{L \times L} \mathbf{I}_{L \times L}\right], \mathbf{W}_{2 L \times L}^{10}=\left[\mathbf{I}_{L \times L} \mathbf{0}_{L \times L}\right]^{T}$, $\mathcal{W}_{L \times 2 L}^{01}=\mathbf{F}_{L} \mathbf{W}_{L \times 2 L}^{01} \mathbf{F}_{2 L}^{-1}, \quad \mathcal{W}_{2 L \times L}^{10}=\mathbf{F}_{2 L} \mathbf{W}_{2 L \times L}^{10} \mathbf{F}_{L}^{-1}$, $\underline{\hat{\mathbf{h}}}_{i}(m)=\mathbf{F}_{L} \hat{\mathbf{h}}_{i}(m), \underline{\hat{\mathbf{h}}}_{i}^{10}(m)=\mathbf{F}_{2 L}\left[\begin{array}{c}\hat{\mathbf{h}}_{i}(m) \\ \mathbf{0}_{L \times 1}\end{array}\right]$ and $\mathcal{D}_{x_{j}}(m)=$ $\mathbf{F}_{2 L}\left[x_{j}(m L-L) x_{j}(m L-L+1) \ldots x_{j}(m L+L-1)\right]^{T}$.

One of the main problems of the NMCFLMS algorithm is that it suffers from a misconvergence problem [7]. Defining the normalized projection misalignment (NPM) [12] as

$$
\eta(n)=\frac{\|\mathbf{h}(n)-\alpha \widehat{\mathbf{h}}(n)\|_{2}^{2}}{\|\mathbf{h}(n)\|_{2}^{2}},
$$

where $\alpha=\left[\mathbf{h}^{T}(n) \widehat{\mathbf{h}}(n)\right] /\left[\widehat{\mathbf{h}}^{T}(n) \widehat{\mathbf{h}}(n)\right]$ and $\mathbf{h}=\left[\mathbf{h}_{1}^{T} \ldots \mathbf{h}_{M}^{T}\right]^{T}$. It can be seen from Fig. 2 that the estimated filter coefficients for the

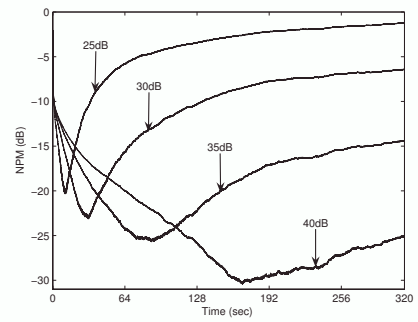

Fig. 2. Misconvergence of the NMCFLMS algorithm at different SNRs.

NMCFLMS algorithm converge first toward the impulse response of the acoustic system after which they then misconverge. Under low signal-to-noise ratio (SNR) conditions, the effect of misconvergence becomes more significant.

\subsection{The extended NMCFLMS algorithm}

The extended NMCFLMS (ext-NMCFLMS) algorithm [8] is proposed to mitigate the misconvergence problem. It minimizes $J(n)$ subject to the constraint that for each $i$ th channel the direct path of estimated channel impulse response is equivalent to the true direct path component, i.e,

$$
\widehat{h}_{i, \mathrm{dp}}(n)=h_{i, \mathrm{dp}}(n),
$$

where 'dp' indicates the tap representing direct path propagation. The cost function and the update equation for ext-NMCFLMS are

$$
\begin{aligned}
& J_{\mathrm{ext}}(n)=\frac{\sum_{i=1}^{M-1} \sum_{j=i+1}^{M} e_{i j}^{2}(n)}{\|\widehat{\mathbf{h}}(n)\|_{2}^{2}}+\beta \sum_{i=1}^{M}\left[h_{i, d p}(n)-\widehat{h}_{i, d p}(n)\right]^{2}, \\
& \underline{\underline{\mathbf{h}}}_{i}^{10}(m+1)=\underline{\hat{\mathbf{h}}}_{i}^{10}(m)-\rho_{\mathrm{E}}\left[\mathcal{P}_{i}(m)+\delta \mathbf{I}_{2 L \times 2 L}\right]^{-1} \sum_{j=1}^{M} \mathcal{D}_{x_{j}}^{*}(m) \underline{\epsilon}_{j i}^{01}(m) \\
& +2 \beta \rho_{\mathrm{E}} \mathbf{F}_{2 L} \mathbf{W}_{2 L \times L}^{10}\left\{\left[h_{i, d p}(m)-\widehat{h}_{i, d p}(m)\right] \widetilde{\mathbf{g}}\right\},
\end{aligned}
$$

where $0<\rho_{E} \leq 1$ is the step-size, $\beta$ is the Lagrange multiplier and the $L \times 1$ vector $\widetilde{\mathbf{g}}=\left[\begin{array}{lll}\mathbf{0}_{1 \times l_{d p}-1} & 1 & \mathbf{0}_{1 \times L-l_{d p}}\end{array}\right]^{T}$ while $\mathcal{P}_{i}(m)$, $\mathcal{D}_{x_{j}}^{*}(m), \underline{\epsilon}_{j i}^{01}(m), \mathbf{F}_{2 L}$ and $\mathbf{W}_{2 L \times L}^{10}$ are defined in subsection 2.1 .

In order to satisfy (7), we require the amplitude and the TDOA between all channels direct path components to be estimated.

\section{THE PROPOSED EXT-NMCFLMS WITH DIRECT-PATH ESTIMATION ALGORITHM}

We propose to estimate both the (a) TDOA and (b) magnitude of the direct path components in order to incorporate these estimates to obtain the proposed ext-NMCFLMS with direct path estimation (ext-NMCFLMS $\mathrm{SPE}_{\mathrm{DE}}$ ) algorithm.

\subsection{The GCC algorithm}

One of the most popular algorithms for TDOA extraction is the generalized cross-correlation (GCC) algorithm [11] which is realized using two prefilters followed by a cross-correlator. The TDOA is obtained by identifying the time-lag corresponding to the highest crosscorrelation between the filtered output of the microphones. The performance of the GCC approach is dependent on the design of the prefilters whose main function is to improve the accuracy of peak detection. We employed the phase transform (PHAT) prefilter which 

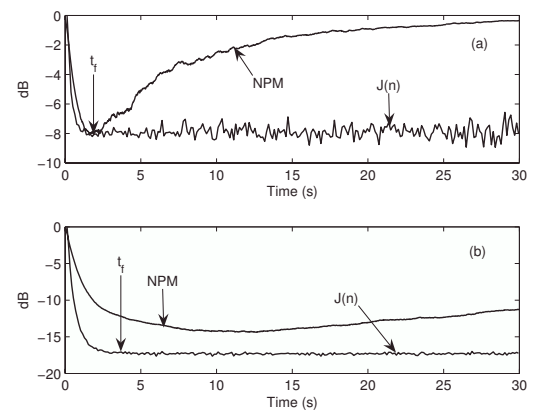

Fig. 3. NPM and Cost function gradient plots for NMCFLMS [6] at (a) SNR $=10 \mathrm{~dB}(\mathrm{~b}) \mathrm{SNR}=20 \mathrm{~dB}, t_{f}$ indicates the flattening point.

has shown to give satisfactory TDOA estimates in the presence of reverberation [13]. The estimated time delay between the $i$ th and $j$ th channel is given by

$$
\widehat{\tau}=\arg \max _{\tau} \widehat{R}(\tau)
$$

where $\widehat{R}(\tau)=\int_{-\infty}^{\infty} G(w) \widehat{P}_{i j}(w) e^{j w \tau} d w, G(w)=1 /\left|\widehat{P}_{i j}(w)\right|$ denotes the PHAT weighting function, $\widehat{P}_{i j}(w)$ denotes the estimated cross-power spectrum and $w$ is the normalized frequency. For reverberant speech, an effective method has been proposed in [14] which performs GCC on the Hilbert envelope of linear prediction (LP) residual of input speech.

\subsection{Online cost function estimation (CFE)}

In order to estimate the magnitude of the direct path components, we propose an online cost function flattening estimation (CFE) method. This method involves estimation of direct path component magnitudes iteratively from the ext-NMCFLMS to monitor the cost function $J(n)$ given by (4) at every iteration so as to estimate the magnitudes of the direct path components. Fig. 3 shows, for two different SNRs, the relationship between $J(n)$ and $\eta(n)$ defined in (6) of the system for the NMCFLMS algorithm. It is clear that the flattening point $t_{f}$ of the cost function $J(n)$ is reached before misconvergence of the algorithm for an SNR as low as $10 \mathrm{~dB}$. Hence the direct path estimates at time $t_{f}$ give us an estimate of the relative magnitudes of the direct path components. The proposed ext-NMCFLMS ${ }_{\text {DPE }}$ algorithm employs online CFE by allowing iterations without direct path substitution following (5) and continually estimating for the flattening point $t_{f}$ using a windowed averaging process on the cost function. After reaching $t_{f}$ the estimated direct path components are frozen and utilized according to (8) during the remaining adaptation of the proposed ext-NMCFLMS ${ }_{\mathrm{DPE}}$ algorithm. By removing the often-used unit-norm constraint we find that the knowledge of the relative magnitudes of direct path components is the only essential requirement to avoid misconvergence in this context and their exact magnitudes are not needed.

\section{SIMULATION RESULTS}

We now present simulation results to study behaviour of the proposed ext-NMCFLMS $S_{\text {DPE }}$ algorithm and compare its performance against the NMCFLMS algorithm [6] and the ext-NMCFLMS algorithm [8] in the context of acoustic room impulse response identification. The dimensions of the room are $(5 \times 4 \times 3) \mathrm{m}$ and impulse responses are generated using the method of images [15]

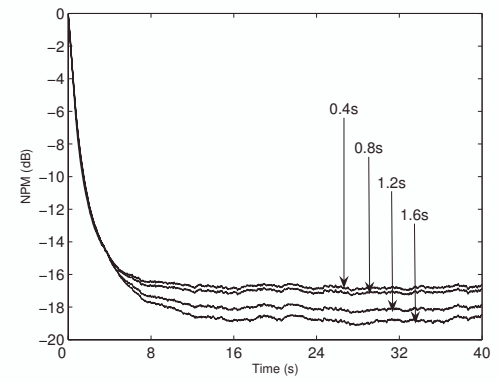

Fig. 4. Effect of freeze of direct path components at different times $=0.4 \mathrm{~s}$, $0.8 \mathrm{~s}, 1.2 \mathrm{~s}$ and $1.6 \mathrm{~s}$ while $t_{f}=1.6 \mathrm{~s}$.

with reverberation time $T_{60}=0.1 \mathrm{~s}$ which are then truncated to length $L=128$. A linear microphone array containing $M=5$ microphones with uniform separation $d=0.2 \mathrm{~m}$ is used. The source and the first microphone are placed at $(1.0,1.5,1.6) \mathrm{m}$ and $(2.0,1.2,1.6) \mathrm{m}$, respectively [9]. Uncorrelated zero-mean WGN is added to the received signal to achieve the SNR specified for each experiment [6]. The sampling frequency is $8 \mathrm{kHz}$ and the SNR is $20 \mathrm{~dB}$ unless otherwise specified. The following parameters are chosen for all simulations [9]: $\lambda=[1-1 /(3 L)]^{L}$ for WGN input, $\lambda=[1-1 /(10 L)]^{L}$ for speech signal input, and $\widehat{\mathbf{h}}_{i}(0)=\left[\begin{array}{llll}1 & 0 & \ldots & 0\end{array}\right]^{T} / \sqrt{M}$.

We first show the effect of estimation errors for the magnitude of the direct path components on the proposed ext-NMCFLMS ${ }_{\mathrm{DPE}}$ algorithm. In Fig. 4, direct-path components are frozen by the CFE method at different time instances. We have used a WGN input with $\mathrm{SNR}=20 \mathrm{~dB}$ and the step-size equal to 0.5 . True time delays for direct-paths have been used. It can be seen that best asymptotic NPM is achieved at freezing time $1.6 \mathrm{~s}$ which corresponds to the flattening point $t_{f}$ of the cost function $J(n)$.

Fig. 5 shows the effect of errors in TDOA estimation $t_{\text {err }}$ of the direct-path components by the GCC method. We have used WGN input with an SNR of $20 \mathrm{~dB}$ while the step-size is 0.5 . This is clear that initial speed of convergence and final asymptotic NPM deteriorate with increasing errors in the delay estimation. This is due to the algorithm missing out on the estimation of the tail samples of the impulse responses. It has been found that the number of missed samples for each channel is equal to the time delay error. Additional experiments have been performed for negative $t_{\text {err }}$ and performance deterioration has not been found. This is due to missing out on bulk zeros at start of acoustic channels which does not effect asymptotic NPM.

Fig. 6 compares the convergence of the proposed algorithm with NMCFLMS [6] and ext-NMCFLMS [8]. In this experiment we employed the CFE method and the GCC algorithm to illustrate the performance of the proposed ext-NMCFLMS ${ }_{\text {DPE }}$ algorithm. We have used WGN as input with an $\mathrm{SNR}=20 \mathrm{~dB}$ while step-sizes are $0.5,0.45$ and 0.7 for ext-NMCFLMS ${ }_{\text {DPE }}, \mathrm{NM}$ CFLMS [6] and ext-NMCFLMS [8] algorithms respectively. The step-sizes have been chosen such that all algorithms reach same asymptotic NPM. True delays for direct-paths have been employed for the ext-NMCFLMS algorithm [8] while we have used GCC with PHAT prefilter to estimate TDOA of direct-paths for the proposed ext-NMCFLMS $S_{\text {DPE }}$ algorithm. The NMCFLMS algorithm misconverges and ext-NMCFLMS converges the fastest. The extNMCFLMS algorithm represents a performance bound for the proposed ext-NMCFLMS $S_{\mathrm{DPE}}$ algorithm as it uses true magnitudes and 


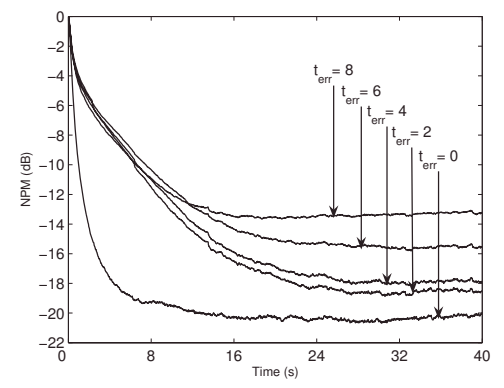

Fig. 5. Effect of error in delay estimation for direct-path component $\mathrm{t}_{\mathrm{err}}=0,2,4,6$ and 8 samples.

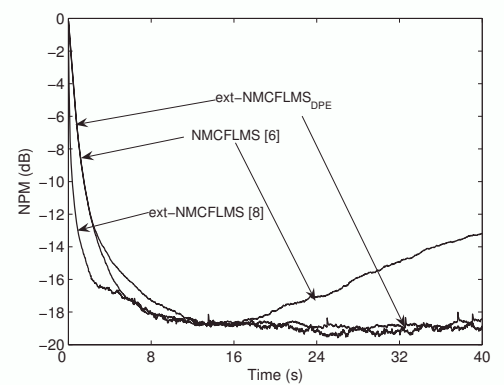

Fig. 6. Comparison of the convergence of the NMCFLMS, est-NMCFLMS and the proposed algorithm with WGN input.

TDOAs. The proposed ext-NMCFLMS DPE $_{\text {algorithm achieves a rate }}$ of convergence comparable with NMCFLMS [6] but an asymptotic performance as good as ext-NMCFLMS [8].

Fig. 7 compares the convergence of the proposed ext-NMCFLMS $S_{D P E}$ algorithm with NMCFLMS [6] and extNMCFLMS [8] algorithms using male speech. We have used an SNR of $20 \mathrm{~dB}$ while step-sizes are $0.05,0.05$ and 0.04 for ext-NMCFLMS $S_{\mathrm{DPE}}$, NMCFLMS and ext-NMCFLMS algorithms respectively. The step-sizes have been chosen such that all algorithms reach same asymptotic NPM. As before, true delays for direct-paths have been employed for ext-NMCFLMS [8] while we have employed GCC with PHAT prefilter of the Hilbert envelope of LP residual of speech [14] to estimate TDOA of direct-paths

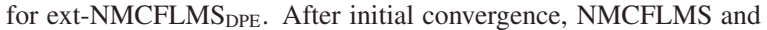
ext-NMCFLMS misconverge whereas ext-NMCFLMS ${ }_{\text {DPE }}$ avoids misconvergence. Other tests have indicated that the proposed ext-NMCFLMS $S_{\mathrm{DPE}}$ algorithm achieves a high rate of convergence which is robust to the presence of noise without misconvergence as compared to NMCFLMS [6] and ext-NMCFLMS [8].

\section{CONCLUSION}

We propose the ext-NMCFLMS $S_{\text {DPE }}$ algorithm for adaptive blind system identification under practical conditions. The proposed algorithm achieves robustness to noise by estimating both the TDOA, using the GCC algorithm, and the magnitude, using the proposed CFE method, of the direct path components. Simulation results for WGN and speech input show the convergence performance of ext-NMCFLMS $\mathrm{DPE}_{\mathrm{DE}}$ is as good as or better than the existing BCI algorithms with the added benefit of robustness to noise.

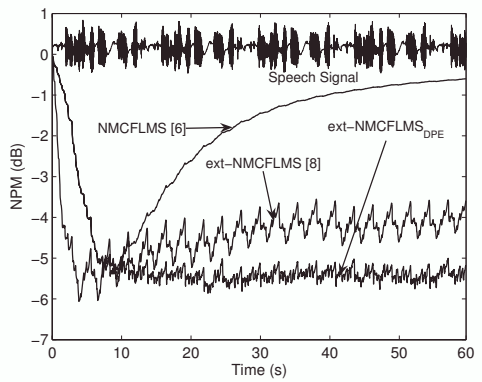

Fig. 7. Comparison of the convergence of the NMCFLMS, est-NMCFLMS and the proposed algorithm with male speech signal input.

\section{REFERENCES}

[1] J. A. Cadzow, "Blind deconvolution via cumulant extrema," IEEE Signal Processing Magazine, vol. 13, no. 6, pp. 24-42, May 1996.

[2] Q-G. Liu, B. Champagne, and P. Kabal, "Room speech dereverberation via minimum-phase and all-pass component processing of multimicrophone signals," in IEEE Pacific Rim Conf. on Communications, Computers, and Signal Processing, May 1995, vol. 1, pp. 571-574.

[3] G. Xu, H. Liu, L. Tong, and T. Kailath, "A least-squares approach to blind channel identification," IEEE Transactions on Signal Processing, vol. 43, no. 12, pp. 2982-2993, Dec. 1995.

[4] E. Moulines, P. Duhamel, J.-F. Cardoso, and S. Mayrargue, "Subspace methods for blind identification of multichannel FIR filters," IEEE Trans. on Signal Processing, vol. 43, no. 2, pp. 516-525, Feb. 1995.

[5] L. Tong and S. Perreau, "Multichannel blind identification: from subspace to maximum likelihood methods," Proc. IEEE, vol. 86, no. 10, pp. 1951-1968, Oct. 1998.

[6] Y. Huang and J. Benesty, "A class of frequency-domain adaptive approaches to blind multichannel identification," IEEE Transactions on Speech and Audio Processing, vol. 51, no. 1, pp. 11-24, Jan. 2003.

[7] Md. Kamrul Hasan and Patrick A. Naylor, "Analyzing effect of noise on LMS-type approaches to blind estimation of SIMO channels: robustness issue," in Proc. 14th European Signal Processing Conf., 2006.

[8] R. Ahmad, A. W. H. Khong, M. K. Hasan, and P. A. Naylor, "The extended normalized multichannel FLMS algorithm for blind channel identification," in Proc. 14th European Signal Processing Conf., 2006.

[9] R. Ahmad, A. W. H. Khong, and P. A. Naylor, "Proportionate frequency domain adaptive algorithms for blind channel identification," in Proc. IEEE Int. Conf. Acoust., Speech, Signal Processing, 2006.

[10] M. K. Hasan, J. Benesty, P. A. Naylor, and D. B. Ward, "Improving robustness of blind adaptive multichannel identification algorithms using constraints," in Proc. 13th European Signal Processing Conf., 2005, vol. 1, pp. 11-14.

[11] G. H. Knapp and G. C. Carter, "The generalized correlation method for estimation of time delay," IEEE Transactions on Acoustics, Speech, and Signal Processing, vol. 24, no. 4, pp. 320-327, Aug. 1976.

[12] D. R. Morgan, J. Benesty, and M. M. Sondhi, "On the evaluation of estimated impulse responses," IEEE Signal Processing Letters, vol. 5, no. 7, pp. 174-176, July 1998.

[13] M. S. Brandstein and H. F. Silverman, "A robust method for speech signal time-delay estimation in reverberant rooms," in Proc. IEEE Int. Conf. Acoust., Speech, Signal Processing, 1997, vol. 1, pp. 375-378.

[14] B. Yegnanarayana, S. R. M. Prasanna, R. Duraiswami, and D. Zotkin, "Processing of reverberant speech for time-delay estimation," IEEE Transactions on Speech and Audio Processing, vol. 13, no. 6, pp. 1110 1118, Nov. 2005.

[15] J. B. Allen and D. A. Berkley, "Image method for efficiently simulating small-room acoustics," J. Acoust. Soc. Amer, vol. 65, no. 4, pp. 943950, Apr. 1979. 